\title{
Editorial
}

\section{The scientist challenge from COVID-19}

Grace Wangge

In this era of the coronavirus diseases 2019 (COVID-19) pandemic, the existence of scientific evidence that may support not only clinical but also regulatory decisions become more prominent. The rapid flow of scientific evidence that emerges since the pandemic, has made us question whether in an emergency situation like this, we might have sacrificed some steps of scientific methods.

Randomized trial is the method currently considered as the most valid and robust to prove the safety and effectiveness of an intervention. This method was originally designed to see the safety and effectiveness of drugs, but in the last decade, this method is also used to assess the effectiveness of medical devices, food, and even recently it is also used to see the effectiveness of a public health programs. This method has even spread beyond the fields of health and medicine and used to evaluate the effectiveness of social intervention programs.

In this edition of the Medical Journal of Indonesia, Diana et $\mathrm{al}^{1}$ presents their research entitled, "A randomized, controlled, cross-over study of the safety and efficacy of super-absorbent diaper for babies with mild-to-moderate diaper rash". In this clinical trial, a seemingly simple product was tested: baby diapers! Diaper rash is one of the most common skin disorders found in babies and in some cases can have serious clinical consequences. For this reason, in this study, Diana et al, ${ }^{1}$ evaluated the safety and efficacy of the use of the latest technology superabsorbent diapers in terms of the incidence of diaper rash compared to standard baby diapers. This study took data from 117 babies who were followed for one month. Before the research was carried out, an explanation to the parents and their consent was asked for, as was the approval of the research implementation from the ethical commission. ${ }^{1}$

In another report, Eliana et $\mathrm{al}^{2}$ described the results of their clinical trial on the provision of dietary supplements that help treat type 2 diabetes mellitus.
Check for updates

This clinical trial used laboratory parameters to assess the clinical response of blood glycemic levels. In addition, the level of subject satisfaction in using this product was also measured using the visual analog scale. Even though it only involved 30 patients, this study still used the principles of good clinical practice and received approval from the ethical commission. ${ }^{2}$

Both of these studies were conducted in the period 2014-2017, and have just been published in our journal in 2020. The peer-review process is perhaps a process that sometimes discourages researchers to disseminate their results. However, this process is necessary as through peer-review, researchers will get inputs regarding the results of their research, and if necessary, how their research can be refined. For this reason, as a matter of fact, dissemination of a study should actually begin at the protocol stage, at least in the form of registering the study in a register. In addition to obtaining initial input, registration of study protocols can help avoid duplication of studies and possibly even opportunities for expansion and collaboration.

Up until early October 2020, in the clinicaltrials.gov, a site for research register based in United States, has 3,507 titles related to COVID-19 in their system. Among them, 1,984 (56.5\%) were interventional trials (clinical trials). In addition, if we look at the randomized clinical trial publications on PubMed in early October 2020, there are at least 68 publications related to COVID-19. This showed that in the last 10 months, global researchers, reviewers, and editors of scientific journals may have conducted faster scientific process than before. Prepandemic, the process of reviewing an article until it can be published in a journal normally takes an average of 9 months to 1 year.

The enthusiasm and increase speed in carrying out this scientific process has resulted in 330 drug candidates and 57 vaccine candidates for COVID-19 that have entered the final phase of clinical trials in humans. ${ }^{3,4}$ Although until now there has been no 
effective definitive or supportive therapy, several drugs and their combinations are considered quite successful in slowing the rate of COVID-19 mortality. This is good news for science, but still needs to be taken with high caution.

Couple months ago, two well-known journals had withdrawn their scientific articles on COVID-19 drugs. Up until now, the site retractionwatch.com (which track retraction scientific publication), has recorded at least 33 retracted articles on COVID-19 in early October 2020. This is actually showing that the scientific process is actually working well and should be continued as it is.

Unfortunately, we observe a trend among some researchers or institutions to skip the peer review process and directly disseminate their research results to general public. Some due to their over enthusiasm to celebrate their eureka moment and the high spirit to help eliminate COVID-19, but some were not. Many of these kinds of information dissemination by scientists were indicated as under influence of external pressures. It is time for us as scientists to demand the importance of an independent scientific environment and be free from any non-scientific pressure. Government should ensure that scientists in their country can work freely and with the highest ethical and scientific standard, free from political and economic demand. Scientific integrity should remain intact!

Apart from that, scientists also need to hone their skills in communicating and disseminating their researchs. In this information age, scientific discussion with other scientists can be done much faster and more convenience through social media and other sharing platforms. However, the public access to scientific information also becomes unlimited and is very vulnerable to be misinterpreted. As scientists, we must be cautious, so that the scientific information we discuss in social media or any open platform is not interpreted differently by readers. We certainly do not want our research will actually triggers hoaxes and disinformation. Ideally, science discussions can be limited to forums and platforms that maintain scientific standards, such as, peer-reviewed journals.

As closing, to answer the challenge from COVID-19 for scientists, we need to use the highest standard of methods in our study and publish them in ways that are in accordance with the principles of science. It is our job to maintain our scientific integrity, despite of many challenges given by the pandemic or any parties that want to take an advantage from the emergency situation.

From Medical Journal of Indonesia; Southeast Asian Ministers of Education-Regional Centre for Food and Nutrition (SEAMEO RECFON)/ Pusat Kajian Gizi Regional Universitas Indonesia (PKGR UI) pISSN: 0853-1773 • elSSN: 2252-8083 https://doi.org/10.13181/mji.ed.205072

Med J Indones. 2020;29:243-4

Corresponding author:

Grace Wangge

E-mail: g.wangge@seameo-recfon.org

\section{REFERENCES}

1. Diana IA, Gondokaryono SP, Sugito TL, Lokanata MD, Agustin $T$, Rahmayunita $G$, et al. A randomized, controlled, cross-over study of the safety and efficacy of superabsorbent diaper for babies with mild-to-moderate diaper rash. Med J Indones. 2020;29(3):283-9.

2. Eliana F, Pranoto BA. A randomized controlled clinical trial of carbohydrate mix-fortified nutrition in type 2 diabetes mellitus patients. Med J Indones. 2020;29(3):275-82.

3. Corum J, Wu KJ, Zimmer C. Coronavirus drug and treatment tracker [Internet]. The New York Times; 2020 [cited 2020 Oct 4]. Available from: https://www.nytimes.com/interactive/2020/ science/coronavirus-drugs-treatments.html.

4. Corum J, Wee SL, Zimmer C. Coronavirus vaccine tracker [Internet]. The New York Times; 2020 [cited 2020 Oct 4]. Available from: https://www.nytimes.com/interactive/2020/ science/coronavirus-vaccine-tracker.html. 